OPEN ACCESS

Edited by:

Yuriy L. Orlov,

Institute of Cytology and Genetics

(RAS), Russia

Reviewed by:

Deepak Singla,

Punjab Agricultural University, India

Hifzur Rahman Ansari,

King Abdullah International Medical

Research Center KAIMRC,

Saudi Arabia

*Correspondence:

Hiroyuki Kurata

kurata@bio.kyutech.ac.jp

${ }^{\dagger}$ These authors have contributed equally to this work

Specialty section:

This article was submitted to Bioinformatics and Computational

Biology,

a section of the journal

Frontiers in Genetics

Received: 25 July 2018 Accepted: 06 February 2019

Published: 05 March 2019

Citation:

Khatun MS, Hasan MM and Kurata H (2019) PreAIP: Computational Prediction of Anti-inflammatory Peptides by Integrating Multiple

Complementary Features.

Front. Genet. 10:129.

doi: 10.3389/fgene.2019.00129

\section{PreAIP: Computational Prediction of Anti-inflammatory Peptides by Integrating Multiple Complementary Features}

\author{
Mst. Shamima Khatun ${ }^{1 \dagger}$, Md. Mehedi Hasan ${ }^{1 \dagger}$ and Hiroyuki Kurata ${ }^{1,2 *}$ \\ ${ }^{1}$ Department of Bioscience and Bioinformatics, Kyushu Institute of Technology, Fukuoka, Japan, ${ }^{2}$ Biomedical Informatics \\ R\&D Center, Kyushu Institute of Technology, Fukuoka, Japan
}

Numerous inflammatory diseases and autoimmune disorders by therapeutic peptides have received substantial consideration; however, the exploration of anti-inflammatory peptides via biological experiments is often a time-consuming and expensive task. The development of novel in silico predictors is desired to classify potential anti-inflammatory peptides prior to in vitro investigation. Herein, an accurate predictor, called PreAIP (Predictor of Anti-Inflammatory Peptides) was developed by integrating multiple complementary features. We systematically investigated different types of features including primary sequence, evolutionary and structural information through a random forest classifier. The final PreAIP model achieved an AUC value of 0.833 in the training dataset via 10-fold cross-validation test, which was better than that of existing models. Moreover, we assessed the performance of the PreAIP with an AUC value of 0.840 on a test dataset to demonstrate that the proposed method outperformed the two existing methods. These results indicated that the PreAIP is an accurate predictor for identifying AIPs and contributes to the development of AIPs therapeutics and biomedical research. The curated datasets and the PreAIP are freely available at http://kurata14.bio.kyutech. ac.jp/PreAIP/.

Keywords: inflammatory disease, anti-inflammatory peptides prediction, feature encoding, feature selection, random forest

\section{INTRODUCTION}

Inflammation responses occur under the normal conditions when tissues are damaged by bacteria, toxins, trauma, heat, or any other reason (Ferrero-Miliani et al., 2007). These responses cause chronic autoimmune and inflammation disorders, including neurodegenerative disease, asthma, psoriasis, cancer, rheumatoid arthritis, diabetes, and multiple sclerosis (Zouki et al., 2000; Steinman et al., 2012; Tabas and Glass, 2013; Patterson et al., 2014; Hernández-Flórez and Valor, 2016). Numerous inflammation mechanisms are crucial for the upkeep of the state of tolerance (Miele et al., 1988; Corrigan et al., 2015). Numerous endogenous peptides recognized through inflammatory reactions function as anti-inflammatory agents can be employed by new therapies for autoimmune and inflammatory illnesses (Gonzalez-Rey et al., 2007; Delgado and Ganea, 2008). The immunotherapeutic aptitude of these anti-inflammatory peptides (AIPs) has various clinical applications such as generation of regulatory $\mathrm{T}$ cells and inhibition of antigen-specific $\mathrm{T}(\mathrm{H}) 1$-driven responses (Delgado and Ganea, 2008). Moreover, certain synthetic AIPs act as effective 
therapeutic agents for autoimmune and inflammatory disorders (Zhao et al., 2016). For instance, chronic adenoidal direction of human amyloid- $\beta$ peptide causes an Alzheimer's disease. Mice models result in compact deposition of amyloid- $\beta$ peptides, which is a pathological marker of Alzheimer's disease, astrocytosis, microgliosis, and neuritic dystrophy in the brain (Boismenu et al., 2002; Gonzalez et al., 2005; Kempuraj et al., 2017). The present therapy for autoimmune and inflammatory disorders involves the use of non-specific anti-inflammatory drugs and other immunosuppressants, which are frequently related to different side effects, such as initiation of a higher possibility of infectious diseases and ineffectiveness alongside inflammatory disorders (Tabas and Glass, 2013).

Notwithstanding the increasing number of experimentally examined AIPs in vivo, the molecular mechanism of AIP specificity remains largely unknown. On the other hand, largescale experimental analysis of AIPs is time-consuming, laborious, and expensive. An alternative, computational approach that provides an accurate and reliable prediction of AIPs is required to complement the experimental efforts and to access the prompt identification of potential AIPs prior to their synthesis. To date, two in silico methods have been proposed to predict AIPs (Gupta et al., 2017; Manavalan et al., 2018). In 2017 Gupta et al. employed hybrid features with a support vector machine (SVM) classifier to develop the AntiInflam predictor (Gupta et al., 2017). Manavalan et al. developed the AlPpred predictor by using the primary sequence encoding features with a random forest (RF) classifier (Manavalan et al., 2018). These two methods used the primary sequence feature information without considering any evolutionary or structural features.

Nonetheless, the performance of the abovementioned existing predictors is not sufficient and remains to be improved. In this study, we have developed an accurate predictor named PreAIP (Predictor of Anti-Inflammatory Peptides) by integrating multiple complementary. We investigated different types sequence features including the primary sequence, evolutionary, and structural through a RF classifier. The PreAIP achieved higher performance on both the training and test datasets than the existing methods. In addition, we obtained valuable insights into the essential sequence patterns of AIPs.

\section{MATERIALS AND METHODS Dataset Collection}

To construct the PreAIP, we collected training and test datasets from a recently published article of the AIPpred (Manavalan et al., 2018) and the IEDB database (Vita et al., 2019). A peptide was considered as anti-inflammatory (positive sample) if the antiinflammatory cytokines of peptides induce any one of IL-10, IL-4, IL-13, IL-22, TGFb, and IFN-a/b in T-cell analyses of mouse and human (Marie et al., 1996; Jin et al., 2014). Meanwhile, the linear peptides for anti-inflammatory cytokines were considered nonAIPs (i.e., negative samples). To solve the overfitting problem of the prediction model, CD-HIT was employed with a sequence identity threshold of 0.8 (Huang et al., 2010). After eliminating redundant peptides, the same training and test samples were retrieved from the AIPpred predictor (Manavalan et al., 2018).
More reliable performance would be achieved by using a more stringent criterion of 0.3 or 0.4 , as executed in (Hasan et al., 2016, 2017a). However, this study did not use such a stringent criterion, because the length of the currently available AIPs is between 4 and 25. If we apply a stringent criterion of $<0.8$, the number of the available AIPs is greatly reduced so that we cannot retrieve the datasets employed by the previous predictor (Manavalan et al., 2018). The collected training dataset results in 1,258 positive and 1,887 negative samples, and the test dataset contains 420 positive and 629 negative samples. All of curated datasets are included in our web server.

\section{Computational Framework}

An overall computational framework of the proposed PreAIP is shown in Figure 1. After collecting the positive and negative AIPs from the AIPpred server (Manavalan et al., 2018), their sequence datasets were transformed into the primary sequence, evolutionary and structural features. We considered polypeptides with 1 to 25 natural amino acids. When the peptide contains less than 25 residues, our scheme provides gaps (-) to the missing residues to compensate a peptide length of 25 . To encode the primary sequence features, we employed two encoding methods of the composition of $k$-spaced amino acid pairs (KSAAP) and AAindex properties. An evolutionary feature was encoded by using the position specific encoding matrix, i.e., profilebased composition $k$-space of amino acid pair (pKSAAP). The structural feature (SF) was encoded by using SPIDER2 (Yang et al., 2017) and PEP2D (http://crdd.osdd.net/raghava/pep2d/) bioinformatics tools. The resulting five types of descriptors were independently put into RF models to produce five consecutive, independent RF prediction scores. Those RF scores were linearly combined using the weight coefficients to obtain the final prediction score. A web server was developed to implement the PreAIP.

\section{Feature Encoding}

The PreAIP was constructed based on a binary classification problem (positive AIPs and negative-AIPs) through RF algorithms. The extraction of a set of relevant features is a crucial step to present a classifier. To keep the generated feature vectors, a high-quality peptide encoding method is necessary. As a substitute of the simple binary representation, we adopted five types of complicated feature encoding methods: AAindex, KSAAP, SPIDER2, PEP2D, and pKSAAP, which are briefly described in the following subsections.

\section{Amino Acid Index Properties}

Numerical physicochemical properties of amino acids exist in the AAindex database (version 9.1) (Kawashima et al., 2008). After assessing different types of AAindex indices, we selected 8 types of high indices (HI) and ordered them from HI1 to HI8 (Table S1). In a peptide sequence with length $L$, a $(L \times 20)$ feature vector was generated through the AAindex encoding.

\section{KSAAP Encoding}

The KSAAP encoding descriptor is widely used in bioinformatics research (Carugo, 2013; Hasan et al., 2018a,b). The procedure of 


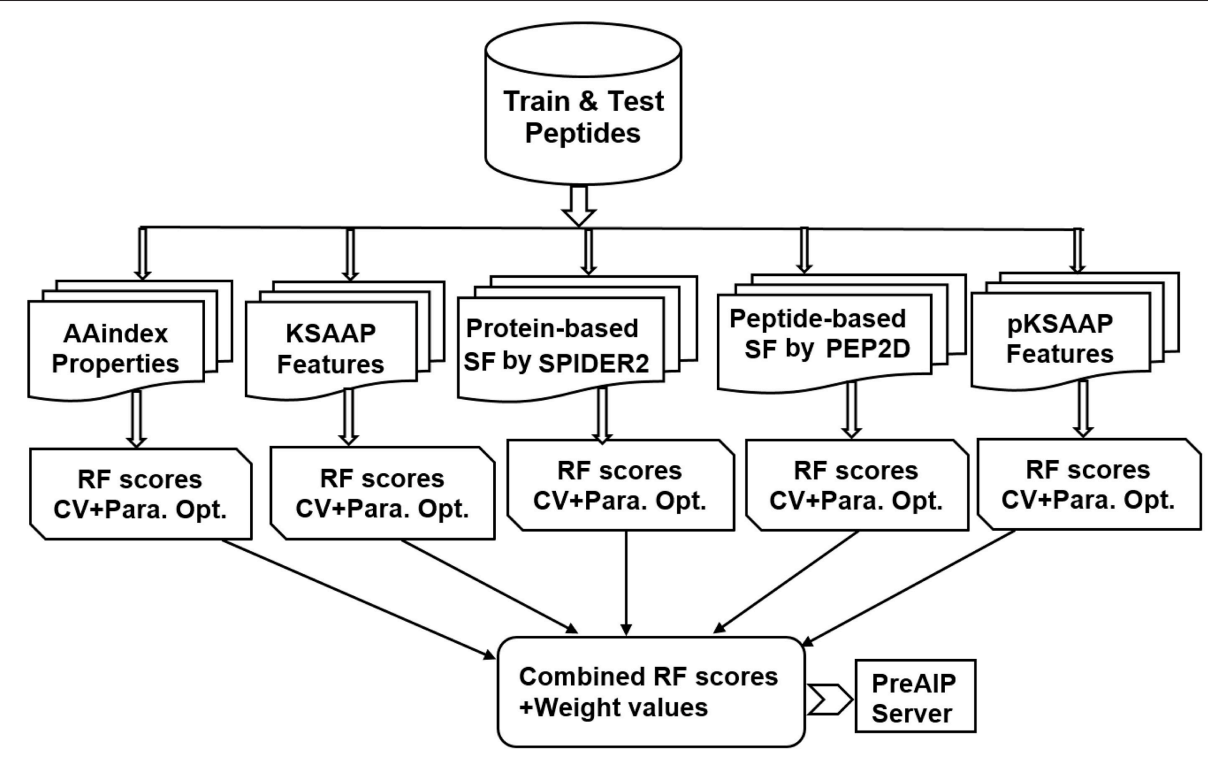

FIGURE 1 | Computational framework of PreAIP.

KSAAP is briefly described as follows. Peptide sequences contain $(20 \times 20)$ types of amino acid pairs (i.e., AA, AC, AD, ..., YY $)_{400}$ for every single $k$, where $k$ denotes the space between two amino acids. The optimal $k_{\max }$ was set to $0-4$ to generate $(20 \times 20 \times$ $5)=2,000$ dimensional feature vectors for each corresponding peptide sequence. Details of the KSAAP encoding method are described elsewhere (Hasan et al., 2015).

\section{Structural Features \\ Protein-Based SF}

The protein-based SF features are generated by the SPIDER2 software that is widely used in bioinformatics research (Yang et al., 2017; López et al., 2018). Three types of features were generated by SPIDER2: accessible surface area (ASA), backbone torsion angles (BTA), and secondary structure (SS). The BTA generated 4-type feature vectors of phi, psi, theta and tau. The SS generated 3-type feature vectors of helix, strand and coil. Totally, 8 -type feature vectors were generated SPIDER2. For each peptide sequence, $(L \times 8)$ dimensional feature vectors were generated, where $L$ was the length of a given AIP.

\section{Peptide-Based SF}

We employed PEP2D to generate a peptide structure prediction feature (http://crdd.osdd.net/raghava/pep2d/). The PEP2D generated three types of probability scores: Helix Prob, Sheet Prob, and Coil Prob. For each peptide sequence, $(L \times 3)$ dimensional feature vectors were generated, where $L$ was the length of a given AIP.

\section{pKSAAP Encoding}

In protein or peptide sequence analysis, the PSSM provides useful evolutionary information. This matrix measures the replacement probability of each residue in a protein with all the residues of the genomic code. The PSSM profile was created by using PSI-BLAST (version of 2.2.26+) against the whole Swiss-Prot NR90 database (version of December 2010) with two default parameters, an e-value cutoff of $1.0 \times 10^{-4}$ and an iteration number of 3 (Hasan et al., 2015). Then, we extracted the feature vectors using the given peptide sequences. After generating the PSSM profile, we generated possible $k$-space pair composition from the PSSM, i.e., pKSAAP, in the same manner as the previous study of protein pupylation site prediction (Hasan et al., 2015). When an optimal $k$-space was between 0 and 4 , a $(5 \times 20 \times 20=$ $2,000)$ dimensional feature vector was generated.

Moreover, we utilized a similarity-search-based tool of BLAST (version of ncbi-blast-2.2.25+) (Altschul et al., 1997; Bhasin and Raghava, 2004) to investigate whether a query peptide belongs to AIPs or not. The BLASTP with an e-value of $1.0 \times 10^{-2}$ was used for the whole Swiss-Prot NR90 database (version of December 2010).

\section{Feature Selection}

To find the top ranking features for predicting AIPs, a wellestablished, supervised method for feature dimensionality reduction, Information Gain (IG) (Azhagusundari and Thanamani, 2013; Huang, 2015; Manavalan et al., 2018), was used through a WEKA package (Frank et al., 2004). A large value of the IG indicates that the corresponding residues have a great impact on prediction performance. The IG processes the decrease in entropy when given information is used to group values of an alternative (class) feature. The entropy of feature $U$ is defined as

$$
H(U)=-\sum_{i} P\left(u_{i}\right) \log _{2}\left(P\left(u_{i}\right)\right)
$$


where $u_{i}$ is a set of values of $U$ and $P\left(u_{i}\right)$ is the prior probability of $u_{i}$. Conditional entropy $H(U / V)$, given another feature $V$, is defined as

$$
H(U \mid V)=-\sum_{j} P\left(v_{j}\right) \sum_{i} P\left(u_{i} \mid v_{j}\right) \log _{2}\left(P\left(u_{i} \mid v_{j}\right)\right)
$$

where $P\left(u_{i} \mid v_{j}\right)$ is the posterior probability of $U$ given by the value $v_{j}$ of $V$. The $I G$ is defined as the decreased entropy calculated by subtracting the conditional entropy of $U$ given by $V$ from the entropy of $U$, as follows.

$$
I G(U \mid V)=H(U)-H(U \mid V)
$$

\section{Random Forest}

The RF is a supervised machine learning algorithm (Breiman, 2001) and is widely used for various biological problems (Manavalan et al., 2017, 2018; Bhadra et al., 2018; Hasan and Kurata, 2018). In brief, the following steps are carried to construct $n$ trees of the RF model. Initially, to obtain a new dataset, $N$ samples are obtained from the training set by random selection with replacement procedures. To get $n$ different datasets this procedure is repeated $n$ times and $n$ decision trees are built based on the $n$ datasets. In this assembling process, for $K$ input features, $k(k<<K)$ features are selected randomly, where $k$ is the constant during construction of the RF. To split the node, a gini impurity criterion is used from the given features. To grow completely, each decision tree is grown without pruning. Afterward getting $n$ decision trees, the class with the most votes is the final prediction (Breiman, 2001). An R package was implemented to train the proposed model (https://cran.r-project. org/web/packages/randomForest/). We set $n$ to 1000 through the 10 -fold cross-validation (CV) test, which is large enough to gain stable prediction.

\section{Other Machine Learning Algorithms}

The performance of the RF was characterized in comparison to three commonly used machine learning algorithms: Naive Bayes (NB) (Lowd, 2005), SVM (Hearst, 1998), and artificial neural network (ANN) (Michalski et al., 2013). We used the $\mathrm{NB}$ and ANN algorithms of the WEKA software (Frank et al., 2004) and the SVM algorithm with a kernel radial basis function (RBF) of the LIBSVM package (https://www.csie.ntu.edu.tw/ cjlin/libsvm/). In the NB algorithm, we set batch size to 1,000 through the 10 -fold CV via the WEKA software. For the ANN algorithm, we considered "MultilayerPerceptron -L $0.3-\mathrm{M} 0.2$ $-\mathrm{N} 500-\mathrm{V}$ OS $0-\mathrm{E} 20-\mathrm{H}$ a" via the WEKA software. To optimize the parameters of the SVM model, the cost and gamma functions were set to 8 and 0.03125 for KSAAP, respectively, via the LIBSVM package. Similarly, the cost and gamma functions were set to 2 and 0.0123 for AAindex, 32 and 0.0625 for pKSAAP, 16 and 0.125 for SPIDER2, and 8 and 0.015625 for PEP2D.

\section{Combined Method}

To make an efficient and robust prediction model, optimization of incorporative feature methods is generally essential. We linearly combined the RF scores of the five encoding methods: AAindex, KSAAP, SPIDER2, PEP2D, and pKSAAP, using the following formula (Hasan et al., 2017b):

$$
\begin{aligned}
\text { Combined } & =w_{1} \times \text { SPIDER } 2+w_{2} \times \mathrm{PEP} 2 \mathrm{D}+w_{3} \times \mathrm{KSAAP} \\
& +w_{4} \times \text { AAindex }+w_{5} \times \mathrm{pKSAAP}
\end{aligned}
$$

where $w_{1}, w_{2}, w_{3}, w_{4}$, and $w_{5}$ are the weight coefficients indicating the strength of the five descriptors; the sum of $w_{1}$, $w_{2}, w_{3}, w_{4}$, and $w_{5}$ is 1 . We adjusted each weight from 0 to 1 with an interval of 0.05 . When $w_{1}, w_{2}, w_{3}, w_{4}$, and $w_{5}$ were 0.00 , $0.00,0.15,0.25$, and 0.6 , respectively, the AUC value on the CV of training dataset was maximal. Therefore, the linear combination of the three successive RF models of KSAAP, AAindex, and pKSAAP was actually "Combined."

\section{Performance Assessment}

To investigate the performance of the PreAIP, the thresholddependent and threshold-independent indices were measured. Using the threshold-dependent indices, four widely used statistical measures denoted as accuracy (Ac) specificity (Sp), sensitivity ( $\mathrm{Sn}$ ), and Matthews correlation coefficient (MCC), respectively, were considered. The four outcomes are presented in the following formulas,

$$
\begin{aligned}
\mathrm{Ac} & =\frac{\mathrm{TP}+\mathrm{TN}}{\mathrm{TP}+\mathrm{FP}+\mathrm{TN}+\mathrm{FN}} \\
\mathrm{Sn} & =\frac{\mathrm{TP}}{\mathrm{TP}+\mathrm{FN}} \\
\mathrm{Sp} & =\frac{\mathrm{TN}}{\mathrm{TN}+\mathrm{FP}} \\
\mathrm{MCC} & =\frac{(\mathrm{TP} \times \mathrm{TN})-(\mathrm{FP} \times \mathrm{FN})}{\sqrt{(\mathrm{TN}+\mathrm{FN}) \times(\mathrm{TP}+\mathrm{FP}) \times(\mathrm{TN}+\mathrm{FP}) \times(\mathrm{TP}+\mathrm{FN})}}
\end{aligned}
$$

where TP exemplifies the number of correctly predicted positive samples; TN the number of correctly predicted negative samples; FP the number of incorrectly predicted positive samples, and FN the number of incorrectly predicted negative samples. Furthermore, we used the receiver operating characteristics (ROC) curve ( $\mathrm{Sn}$ vs. 1-Sp plot) to evaluate the area under the ROC curve (AUC) of the threshold-independent parameter (Centor, 1991; Gribskov and Robinson, 1996).

Since the balance between the correctly predicted AIPs and non-AIPs is critically responsible for accurate prediction, Sp and $\mathrm{Sn}$ are intuitive, intelligible measures. Typically, high $\mathrm{Sp}$ decreases Sn. In this study, the prediction performance of the PreAIP for the training dataset was evaluated with a stepwise change in Sp. We calculated Sn, Ac, and MCC at high (0.903), moderate (0.801) and low (0.709) levels of Sp. These three levels of Sp were given by setting the high $(0.468)$, moderate $(0.388)$, and low (0.342) thresholds of the RF score. In the same manner, we measured the performance of the individual encoding scheme of KSAAP, AAindex, SPIDER2, PEP2D, and pKSAAP at each level of Sp. When the same threshold values of the RF score were applied to prediction of the test dataset, the high, moderate 
and low levels of Sp were calculated as $0.871,0.747$, and 0.636 , respectively.

To assess the performance of the PreAIP using the measures of Ac, Sp, Sn, MCC, and AUC, a 10-fold CV test was used. For the 10 -fold CV, original training samples were randomly and equally picked up into 10 subclasses. Among 10 subclasses, one subclass was singled out as the test sample, and the remaining 9 subclasses were considered as the training sample. Then we computed all performance measures for each predictor. We repeated this procedure 10 times by changing the training and test samples. Eventually, we calculated the average value of each performance measure for each predictor.

\section{RESULTS AND DISCUSSION}

\section{Sequence Preference Analysis of AIPs}

To investigate the amino acid preference of positive and negative AIPs, we performed sequence compositional preference analysis using the amino acids from the 1 to $15 \mathrm{~N}$-terminal residues of training sets. The length of the AIPs ranged between 4 and 25 amino acid residues in this study. The average length of AIPs was 15 amino acids. Since Ialenti et al. suggested that the AIP activity is located in the $\mathrm{N}$-terminal region of the molecule (Ialenti et al., 2001), we investigated the 1 to $15 \mathrm{~N}$-terminal amino acids by the sequence compositional preference analysis. A non-existing residue was coded by "O" to fill the corresponding position of the AIPs.

At first, we submitted the 1 to $15 \mathrm{~N}$-terminal amino acids of positive and negative AIPs to the sample logo online server (http://www.twosamplelogo.org/) to generate the sequence logo representations (Figure 2). The height for each amino acid was in proportion to the percentage of positive (over-represented) or negative (under-represented) peptides. The logos were scaled according to their statistical significance threshold of $p<0.05$ by Welch's $t$-test. Leucine (L) at positions $5,7,10,11$, and 15, cysteine (C) at position 7 and 10, isoleucine (I) at positions 2 and 7 , arginine $(\mathrm{R})$ at position 5 , phenylalanine $(\mathrm{F})$ at position 8 , and lysine $(\mathrm{K})$ at position 15 were significantly overrepresented compared with other amino acids, while aspartic acid (D) at positions $4,5,10,13$, and 15 , threonine (T) at positions 3 and 7 , valine $(\mathrm{V})$ at position 15 were significantly underrepresented. In addition, tyrosine (Y) at positions 4 and 5 was overrepresented, while $\mathrm{Y}$ at positions 5 and 10 underrepresented. These results suggested that positive and negative AIPs are significantly different.

Secondly, we examined the evolutionary conservation features of the PreAIP using the average PSSM value (APV) for each amino acid within 1 to15 N-terminal amino acids of AIPs. The evolutionary conservation information of APV of both the positive and negative AIPs is illustrated in Figure 3. Some of amino acid positions of positive and negative AIPs showed significantly different scores. Furthermore, a nonparametric Kruskal-Wallis (KW) test was used to examine whether positive and negative AIPs were significantly dissimilar. The $p$-values were calculated and corrected by the Bonferroni test (Table S2).

Thirdly, we examined the AAindex encoding features of PreAIP. Eight types of informative amino acid indices were

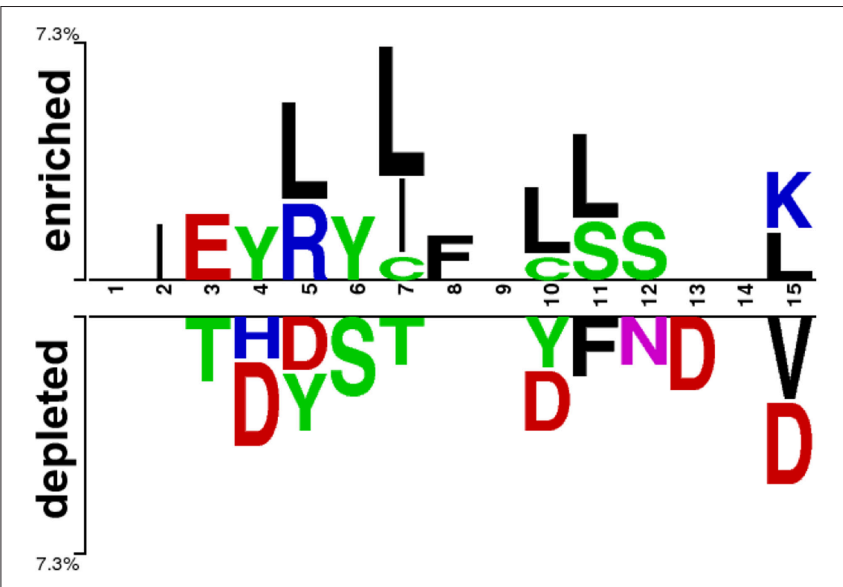

FIGURE 2 | Sequence logo representation of positive and negative AIPs. The upper portion (enriched) is represented by positive AIPs, while lower portion (depleted) negative AIPs. The statistically significant local sequence within the $\mathrm{N}$-terminal 15-residues of AIPs was plotted with $p<0.05$ by Welch's $t$-test.

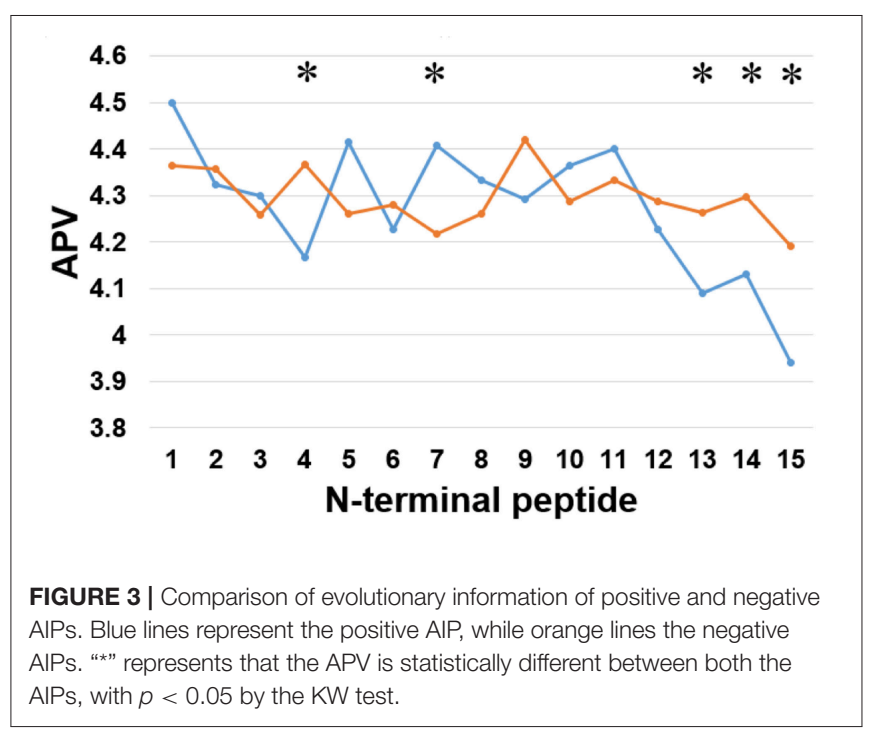

used and named HI1 to HI8 as the input feature vectors from the AAindex database. We examined these HI amino acid properties of both the positive and negative AIPs. As illustrated in Figure 4, the average values of the eight indices were renamed as AVHI1 to AVHI8. These indices represented the amino acid compositions of intracellular proteins. Some of the AIPs had distinct amino acid compositions in the eight high-quality amino acid indices between two samples of AIPs (Figure 4). The KW test was used to examine whether two samples of AIPs were significantly dissimilar with respect to the eight $\mathrm{HI}$ properties. The $p$-values were calculated and corrected by the Bonferroni test (Table S3). Significantly different AAindex values with $p$-value $<0.05$ appeared at some positions of AIPs, as marked with "**" in Figure 4.

Finally, we examined the difference in 8 types of SFs by SPIDER2 between the positive and negative AIPs, as shown in 

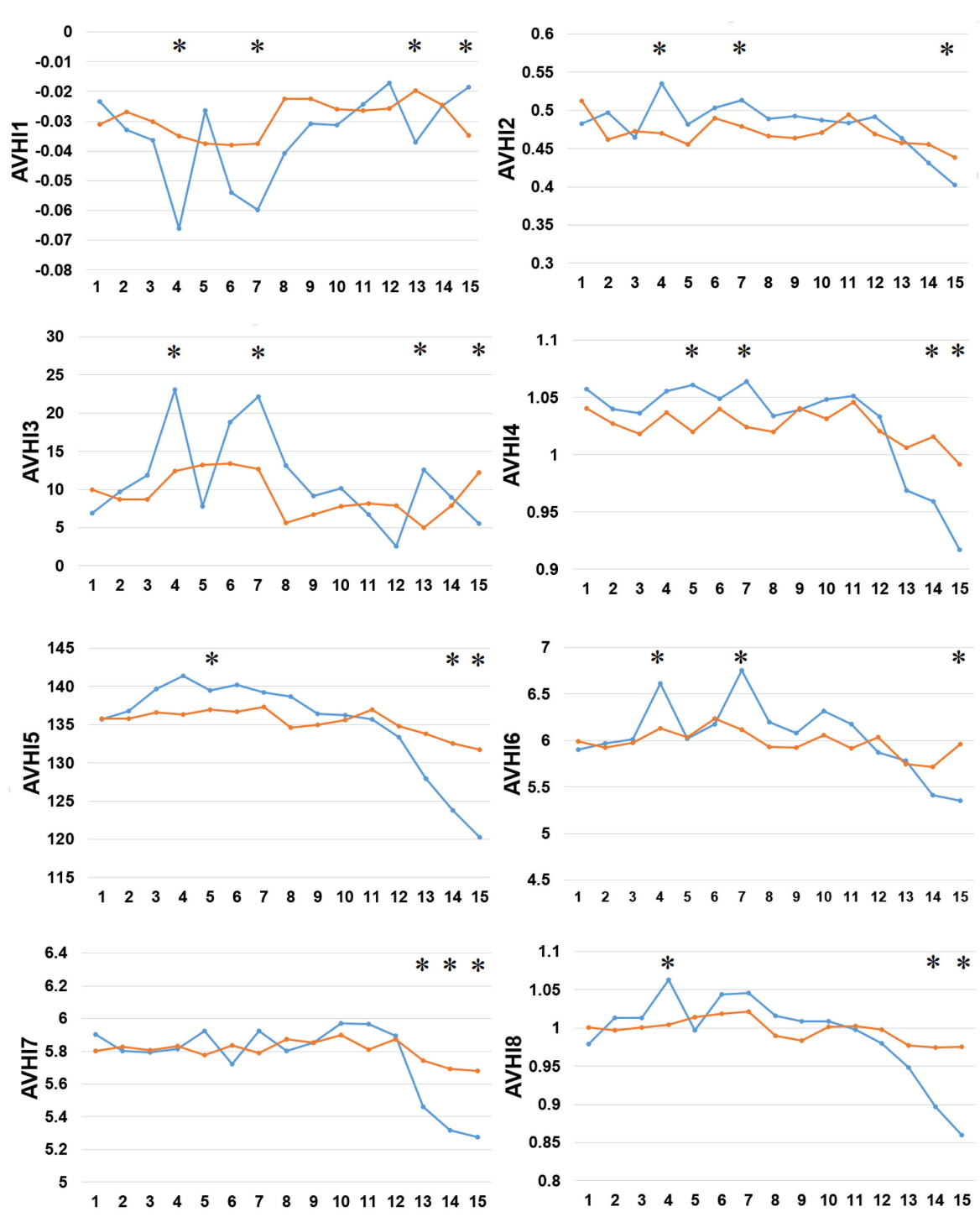

FIGURE 4 | Comparison of eight high-quality amino acid indices between two samples of AIPs. The eight high-quality amino acid indices from HI1 to HI8 are placed at the centers of eight amino acid index clusters, which indicate high residue propensities of AAindex. The row represents the $\mathrm{N}$-terminal peptide, while the blue lines signify the positive AIP and the orange lines the negative AIPs. "*” represents that the amino acid indices are statistically different between both the samples with $p<$ 0.05 by the KW test.

Figure 5. We calculated the average value of 8 types of SFs for SPIDER2: ASA, phi, psi, theta, tau, coil, stand, and helix of both the positive and negative AIPs. The average features were represented as AVAS, AVPhi, AVPsi, AVThe, AVTau, AVCoil, AVSta, and AVHel (Figure 5). We plotted these average values of SFs with respect to the 1-15 N-terminal AIPs. Distinguished differences were observed between the positive and negative samples of AIPs. The KW test was employed to examine whether two sample of AIPs were significantly dissimilar among the eight SFs. The $p$-values were calculated and corrected by the Bonferroni test (Table S4). Significantly different SFs were perceived at some positions of AIPs, with a $p$-value $<0.05$, as indicated with "*” in Figure 5.

The above analysis of residue preference between the positive and negative AIPs suggested that the combination of the primary sequence, evolutionary, and structural amino acid occurrences achieves a precise prediction.

\section{Overall Prediction Performance of PreAIP}

The selected five descriptors (AAindex, KSAAP, SPIDER2, PEP2D, and pKSAAP) were separately used for prediction of AIPs. Optimization of multiple encoded features is generally essential in the training model to reduce dimensionality while retaining the significant feature. To achieve this, we performed multiple rounds of experiments to select appropriate feature vectors using the IG feature selection via 10 -fold $\mathrm{CV}$ test on training set; however, it turned out that the IG feature selection did not improve prediction performance. Thus, the IG feature was used to collect significant features and for interpreting a superiority of KSAAP encoding. 

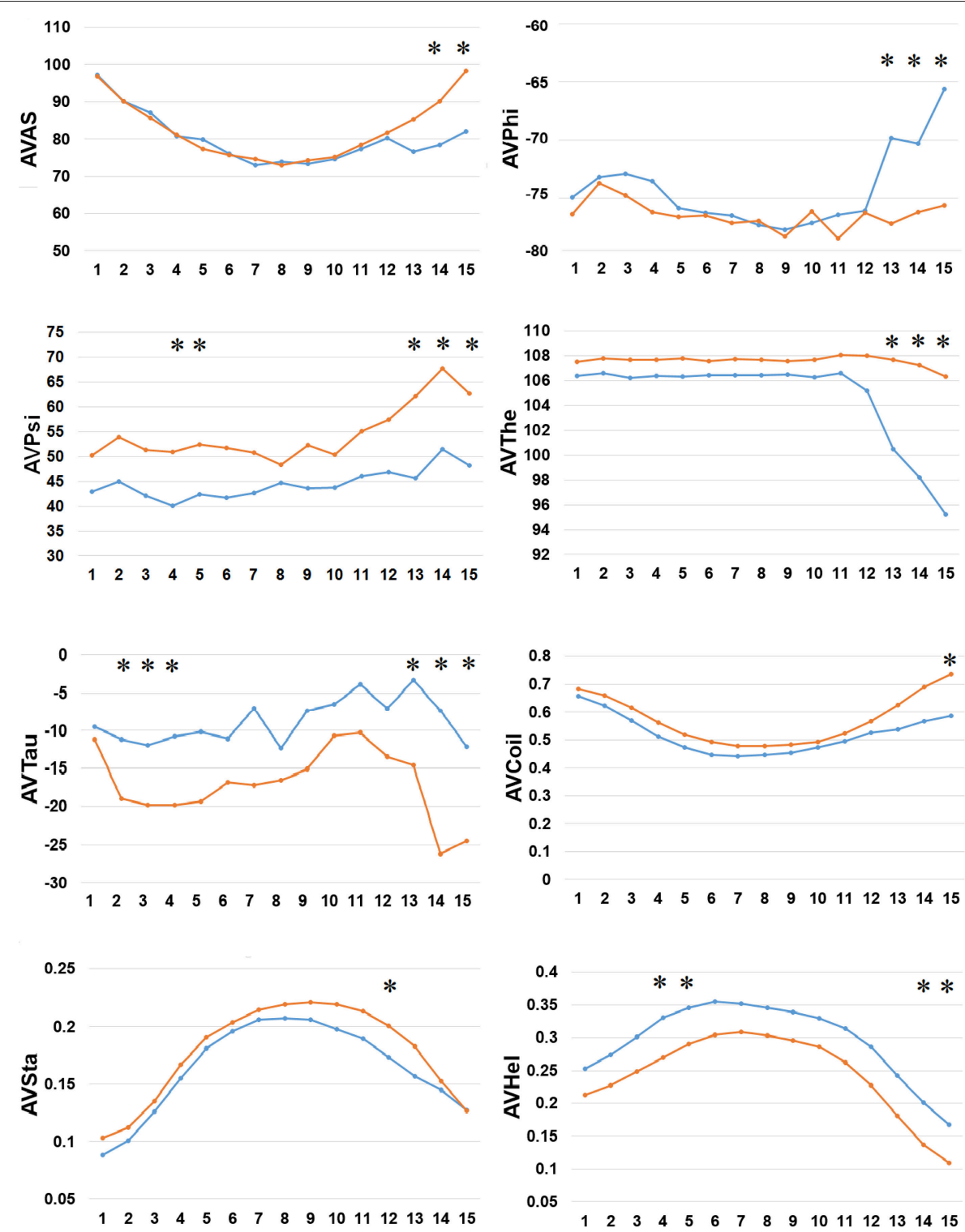

FIGURE 5 | Comparison of 8 types of the SFs by SPIDER2 between positive and negative AIPs. The row represents the N-terminal peptide, while the blue lines signify the positive AIPs and the orange lines the negative AIPs. "*" represents that the SFs are statistically different between both the samples with $p<0.05$ by the KW test.

We accessed the performances of the training model of five successive encoding methods of AAindex, KSAAP, SPIDER2, PEP2D, and pKSAAP through a 10 -fold CV test using the RF classifier. The prediction results by each of five encoding features and the "Combined features" are shown in Figure 6A. The AUCs of AAindex, KSAAP, SPIDER2, PEP2D, and pKSAAP were $0.774,0.813,0.739,0.734$, and 0.789 , respectively. The KSAAP performed best for the 5 single encoding approaches in terms of Sn, MCC and AUC (Table 1). The "Combined features" (PreAIP) showed better performance with an AUC of 0.833 than any other single feature. It is noted that "Combined features" means a linear combination of the RF scores (Materials and Methods). Moreover, the PreAIP presented the highest AUC value (0.840) in the test dataset (Figure 6B). The performance of PreAIP was effective and reasonable for all the tested cases (Figure 6) and was best in the AIP prediction.

To present the known AIPs in the training dataset, we used BLAST to search the (weak) homologs, and ranked them to obtain the best hit e-value (Bhasin and Raghava, 2004). Total 256 positive and 397 negative hits were found out of 1,258 positive and 1,887 negative samples by BLASTP with an evalue of $1.0 \times 10^{-2}$. The reduced numbers of the samples may be due to the peptide length of 5-25. Then, we measured the BLAST performances through 10 -fold $\mathrm{CV}$ test. The prediction performances of Sp, Sn, Ac, MCC, and AUC were 0.752, 0.269, $0.563,0.159$, and 0.632 , respectively, which were lower than those 

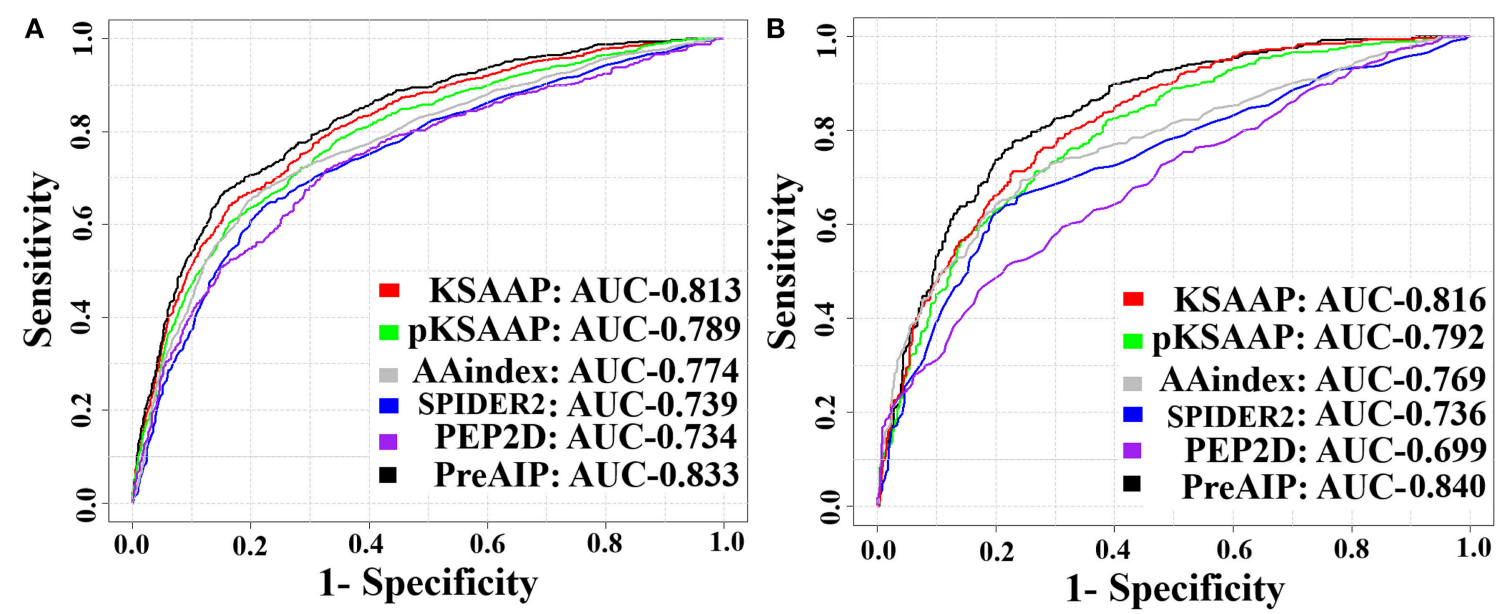

FIGURE 6 | ROC curves of the various prediction models. (A) 10-fold CV test on a training dataset and (B) test dataset. The PreAIP combined the KSAAP, pKSAAP, and AAindex methods. High AUC values show accurate performance.

TABLE 1 | AUC values for prediction performance of the training dataset by 10-fold CV test.

\begin{tabular}{lcccccc}
\hline Methods & Sp & Sn & Ac & MCC & AUC & $\boldsymbol{p}$-value \\
\hline pKSAAP & 0.798 & 0.647 & 0.738 & 0.450 & 0.789 & 0.017 \\
AAindex & 0.795 & 0.644 & 0.735 & 0.448 & 0.774 & 0.012 \\
SPIDER2 & 0.765 & 0.434 & 0.633 & 0.235 & 0.739 & 0.004 \\
PEP2D & 0.769 & 0.411 & 0.629 & 0.219 & 0.734 & 0.004 \\
KSAAP & 0.805 & 0.656 & 0.745 & 0.463 & 0.813 & 0.118 \\
PreAIP* & 0.806 & 0.709 & 0.767 & 0.508 & 0.833 & \\
\hline
\end{tabular}

${ }^{*}$ PreAIP is the linear combination of the RF scores estimated by SPIDER2, PEP2D, KSAAP, AAindex, and PKSAAP encoding schemes and their weight coefficients are 0.00, 0.00, $0.15,0.25$, and 0.6 , respectively. A p-value was computed based on the final model of AUC values by using a Wilcoxson matched-pair signed test.

by the other sequence encoding-based models. Therefore, we did not consider BLAST for final prediction.

In addition, we found that KSAAP performed best for all the five single encoding methods. To investigate the most significant residue of the KSAAP method, the top 20 amino acid pairs of AIPs were examined through the IG feature selection. The top 20 significant residue pair scores and their corresponding positions are listed in Table S5. These significant features are also presented using a radar diagram (Figure 7A). For example, the feature sequence motif " $\mathrm{L} \times \mathrm{L}$," which is represented by 1 spaced residue pair of "LL," is the most important residue pair, where " $x$ " stands for any amino acid. The feature " $L \times \times \times L$ " represented the second enriched motif surrounding positive samples of AIPs. Similarly, the feature "LL," which represents a 0 -spaced residue pair of "LL," is important and enriched in the negative samples AIPs. Similarly, to keep other $k$-space amino acid pairs from KSAAP, the same exemplification was employed. Residue preference analysis demonstrated that "L," "Y," "C," "D," and "I" residues frequently appear for AIPs (Figures 2, 7A). These residues are expected to play a key role in the recognition of AIPs. To characterize the top 20 KSAAP-specific features, we compared the numbers of positive and negative AIPs. Figure $7 \mathbf{B}$ showed the top 20 average value of feature scores (AVFS) by the IG. The average of top 20 features was significantly different between two samples of AIPs with $p<0.05$, suggesting the effectiveness of the KSAAP encoding. The significant residue pair scores are listed in Table S5, which provides some insights into the sequence patterns of the AIPs. They deserve further experimental validation.

\section{Comparison of PreAIP With Existing Predictors Using Test Dataset}

We evaluated the performances of PreAIP along with that of existing predictors on the test dataset. We submitted the test set to the AIPpred (Manavalan et al., 2018) and AntiInflam (Gupta et al., 2017) servers to assess the performance. It is noted that AntiInflam server provides different thresholds values. We used two threshold values of -0.3 and 0.5 and renamed as less accurate (LA) and more accurate (MA) models (Gupta et al., 2017), respectively. The AIPpred represents the state-of-the-art predictor available. The average performances of the LA, MA, AIPpred, and PreAIP are illustrated in the Table 2. The LA showed the highest Sp (0.892) with the lowest Sn (0.258), MCC (0.197), and AUC (0.647) for all the predictors. The PreAIP with the high threshold presented much higher Sn (0.618) Ac (0.770), MCC (0.512), and AUC (0.840) than LA, while it provided Sp (0.871) comparable to LA. The PreAIP with the low threshold showed the highest Sn (0.863), while keeping Sp, Ac, MCC, and AUC at a high level. While the AIPpred presented considerably high values to all the measures of Sp, Sn, Ac, MCC, and AUC, the PreAIP with the moderate threshold outperformed the AIPpred, presenting well-balanced, high prediction performances. The PreAIP performance improvement was found distinct on the test dataset by the Wilcoxson matched-pair signed test, demonstrating its ability to predict unseen peptides.

\section{Comparison of PreAIP With AIPpred Using Training Dataset}

We compared the performance of the proposed PreAIP with the AIPpred using the same training dataset. In this study, the same dataset as the AIPpred set was used to make a fair comparison 


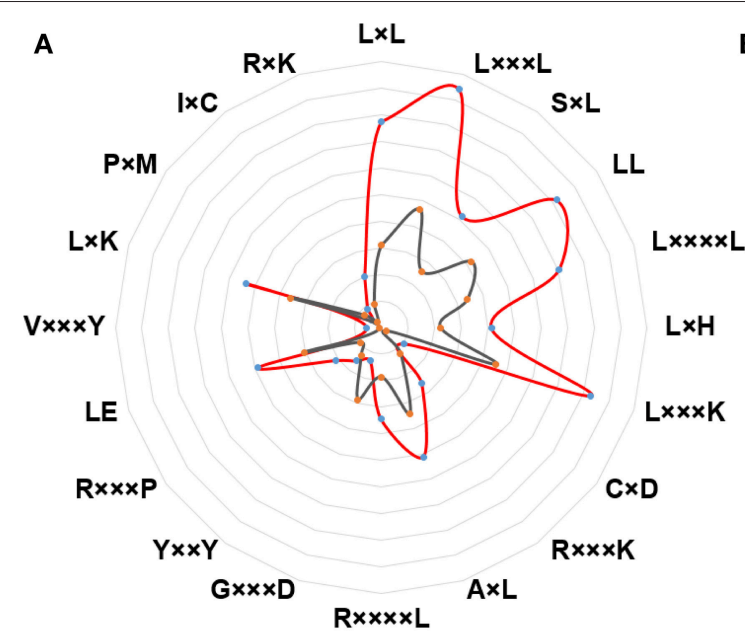

B

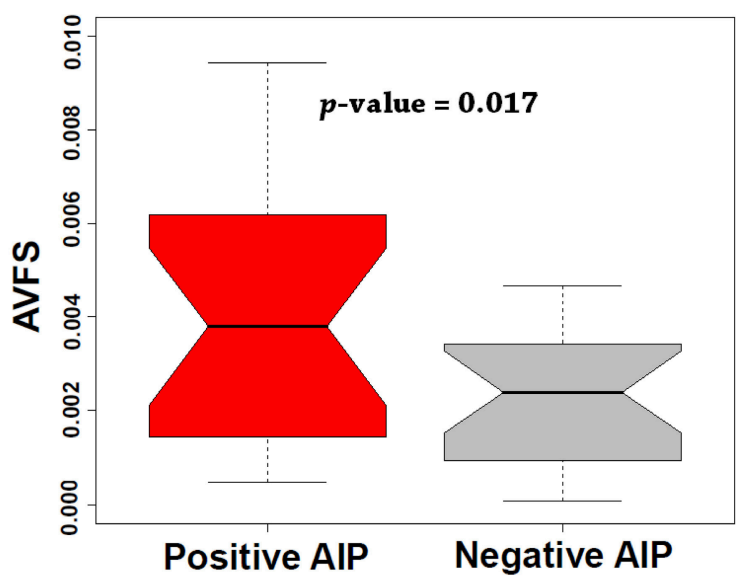

Positive sample Negative sample

FIGURE 7 | Top 20 amino acid pairs selected by the IG feature of the KSAAP method. (A) The radar diagram is represented by the composition of each amino acid pair whose length is proportional to the composition of KSAAP features. (B) Box plot shows the top 20 average value of feature scores (AVFS) by the IG. Red color denotes the positive AIPs, while gray color denotes the negative AIPs. The $p$-value is computed by two-sample $t$-test.

TABLE 2 | Performance comparison with exiting predictors using test dataset.

\begin{tabular}{llllllll}
\hline Predictor & \multicolumn{2}{l}{ Threshold $\mathbf{S p}$} & Sn & Ac & MCC & AUC & $\boldsymbol{p}$-value \\
\hline Antilnflam $(\mathrm{LA})$ & -0.3 & 0.892 & 0.258 & 0.638 & 0.197 & 0.647 & $<0.001$ \\
Antilnflam $(\mathrm{MA})$ & 0.5 & 0.417 & 0.786 & 0.565 & 0.210 & 0.706 & $<0.001$ \\
AlPpred & Server & 0.746 & 0.741 & 0.744 & 0.479 & 0.813 & 0.039 \\
PreAlP & High & 0.871 & 0.618 & 0.770 & 0.512 & 0.840 & \\
& Moderate & 0.747 & 0.784 & 0.762 & 0.522 & 0.840 & \\
& Low & 0.636 & 0.863 & 0.727 & 0.492 & 0.840 &
\end{tabular}

A p-value was computed based on AUC values by using a Wilcoxson matched-pair signed test and $p<0.05$ indicates a statistically significant difference between the proposed PreAIP and each selected method. The performances of Antilnflam LA and MA methods were computed using default threshold (server) values of -0.3 and 0.5 , respectively. The AlPpred threshold was the same as given by its server.

for prediction performance of AIPs. As shown in Table 3, the PreAIP achieved a better performance than the AIPpred in terms of Ac, Sp, Sn, MCC, and AUC. The AUC value was nearly 3\% higher than the AIPpred predictor. The PreAIP performance (AUC) improvement over the AIPpred was demonstrated on the training set by the Wilcoxson matched-pair signed test (Table 3).

\section{Comparison of Different Machine Learning Algorithms}

The performance of the RF was compared to the three widely used machine learning algorithms, NB, SVM, and ANN by using the same training datasets and features, as shown in Table 4. The AUC values of the prediction by the five algorithms were calculated by a 10-fold CV test, while using the SPIDER2, PEP2D, AAindex, KSAAP, and pKSAAP encodings and their combined method. The RF provided higher AUC than any other algorithms for all the encoding methods and their combined method.
TABLE 3 | Performance comparison of PreAIP with AlPpred using training dataset.

\begin{tabular}{|c|c|c|c|c|c|c|c|}
\hline Method & sThreshold & $\mathrm{Sp}$ & Sn & Ac & MCC & AUC & $p$-value \\
\hline AlPpred & $\begin{array}{l}\text { Default } \\
\text { given in the } \\
\text { server }\end{array}$ & 0.711 & 0.758 & 0.730 & 0.460 & 0.801 & 0.034 \\
\hline \multirow[t]{3}{*}{ PreAIP } & High & 0.903 & 0.632 & 0.795 & 0.566 & 0.833 & \\
\hline & Moderate & 0.801 & 0.719 & 0.768 & 0.520 & 0.833 & \\
\hline & Low & 0.709 & 0.784 & 0.739 & 0.484 & 0.833 & \\
\hline
\end{tabular}

A p-value was computed based on AUC values by using a Wilcoxson matched-pair signed test and $p<0.05$ indicates a statistically significant difference between the proposed PreAIP and AIPpred.

\section{The Effect of Peptide Redundancy on the Predictive Model}

The peptide redundancy may lead to the overestimation on the predictive performance. Therefore, we performed the CD-HIT with 60\% identity cutoff at the peptide level (Huang et al., 2010). After removing the $60 \%$ sequence redundancy, we re-assembled a training dataset that contained 1,098 positive and 1,226 negative samples, and the test dataset that contained 308 positive and 275 negative samples. While the overall performance (AUC = 0.821) of the PreAIP by the 10-fold CV test decreased slightly (Table S6), the PreAIP could still achieve the best performance on the independent testing dataset (Figure S1). The PreAIP achieved 6 and $8 \%$ higher AUC values than the AntiInflam and the AIPpred, respectively, demonstrating that the PreAIP with the $60 \%$ peptide redundancy removal provides a stable or competitive performance compared with the other predictors, as well as the $80 \%$ peptide redundancy removal. 
TABLE 4 | AUC values of AIP prediction by different machine learning algorithms based on a 10 -fold $\mathrm{CV}$ test.

\begin{tabular}{lcccccc}
\hline Algorithms & SPIDER2 & PEP2D & AAindex & KSAAP & pKSAAP & Combined \\
\hline RF & 0.739 & 0.734 & 0.774 & 0.813 & 0.789 & 0.833 \\
NB & 0.659 & 0.655 & 0.707 & 0.729 & 0.717 & 0.736 \\
SVM & 0.698 & 0.677 & 0.738 & 0.766 & 0.749 & 0.779 \\
ANN & 0.662 & 0.649 & 0.716 & 0.741 & 0.736 & 0.753 \\
\hline
\end{tabular}

"Combined" indicates that the performance of the optimized combined features. The combined score of RF was given as the sum of the five SPIDER2, PEP2D, AAindex, KSAAP, and PKSAAP features with weight values of $0.00,0.00,0.15,0.25$, and 0.6 respectively. In the same way, the weight values of NB, SVM, and ANN were given as (0.00, 0.00, 0.10, 0.35, and 0.55), (0.00, 0.00, 0.22, 0.45, and 0.33), and (0.00, 0.00, $0.18,0.5$, and 0.32 ), respectively.

\section{Advantages of PreAIP}

In theoretical viewpoints, comparison of the proposed PreAIP with existing predictors is summarized: (1) The PreAIP investigated the primary sequence, physicochemical properties, structural, and evolutionary features, although the AIPpred and AntiInflam predictors used only primary sequence encoding method. For instance, in AntiInflam method (Gupta et al., 2017), studied hybrid features based on primary sequence encoding schemes such as amino acid composition (AAC), dipeptide composition (DPC), and tripeptide composition with SVM algorithm. The AIPpred (Manavalan et al., 2018) studied individual composition (AAC, AAindex, DPC, and chaintransition-composition) through multiple machine learning algorithms. (2) Since existing prediction tools did not control the Sp level, users cannot understand which AIP is highly positive or negative from their servers. On the other hand, the PreAIP controlled Sp at high, moderate and low levels by changing the threshold of the RF scores, based on 10-fold CV test results. A limitation of the PreAIP is that the employed dataset is still small, but we believe that the dataset will grow to enable intensive identification of AIPs. In addition, the calculation speed remains to be improved. The processing time of the PreAIP was $<3 \mathrm{~min}$ for one peptide sequence, where the generation of PSSM profiles requires a long time.

\section{Server of PreAIP}

A web server of the PreAIP has been developed and publically accessible at http://kuratal4.bio.kyutech.ac.jp/PreAIP/. The web application was implemented by programming languages of Java scripts, Perl, R, CGI scripts, PHP, and HTML. After submitting a query sequence to the server, it generates consecutive feature vectors. Then, the server optimizes the performances through

\section{REFERENCES}

Altschul, S. F., Madden, T. L., Schäffer, A. A., Zhang, J., Zhang, Z., Miller, W., et al. (1997). Gapped BLAST and PSI-BLAST: a new generation of protein database search programs. Nucleic Acids Res. 25, 3389-3402. doi: 10.1093/nar/25. 17.3389

Azhagusundari, B., and Thanamani, A. S. (2013). Feature selection based on information gain. Int. J. Innov. Technol. Explor. Eng. 2, 2278-3075.

Bhadra, P., Yan, J., Li, J., Fong, S., and Siu, S. W. I. (2018). AmPEP: sequence-based prediction of antimicrobial peptides using distribution
RFs. After completing the submission job, the server returns the result in the output webpage which consists of the job ID and probability scores of the predicted AIPs in a tabular form. A user gets a job ID like "2018032900067" and can save this ID for a future query. The server stores this job ID for one month. The input peptide sequence must be in the FASTA format. Each of the 20 types of standard amino acids must be written as one uppercase letter. See the test example on the server. The length of AIP sequence was limited from 1 to 25 . If users submit 200 amino acids, the PreAIP takes first 1-25 residues to analyze. When the peptide contains less than 25 residues, the PreAIP provides gaps $(-)$ to the missing residues to compensate a peptide length of 25 .

\section{CONCLUSIONS}

We have designed an accurate and efficient computational predictor for identifying potential AIPs. It outperforms the existing methods and is effective in understanding some mechanisms of AIP identification. An IG-based feature selection method was carried out to suggest sequence motifs of AIPs from KSAAP encoding. A user-friendly web-server was developed and freely available for academic users.

\section{AUTHOR CONTRIBUTIONS}

$\mathrm{MK}, \mathrm{MH}$, and HK conceived and designed the study. MK and MH collected data and performed the analyses. MH, MK, and HK wrote the manuscript. All authors discussed the prediction results and commented on the manuscript.

\section{ACKNOWLEDGMENTS}

This work was supported by the Grant-in-Aid for Challenging Exploratory Research with JSPS KAKENHI Grant Number 17K20009. This research is partially supported by the developing key technologies for discovering and manufacturing pharmaceuticals used for next-generation treatments and diagnoses both from the Ministry of Economy, Trade and Industry, Japan (METI) and from Japan Agency for Medical Research and Development (AMED).

\section{SUPPLEMENTARY MATERIAL}

The Supplementary Material for this article can be found online at: https://www.frontiersin.org/articles/10.3389/fgene. 2019.00129/full\#supplementary-material

patterns of amino acid properties and random forest. Sci. Rep. 8:1697. doi: 10.1038/s41598-018-19752-w

Bhasin, M., and Raghava, G. P. (2004). GPCRpred: an SVM-based method for prediction of families and subfamilies of G-protein coupled receptors. Nucleic Acids Res. 32, W383- W389. doi: 10.1093/nar/gkh416

Boismenu, R., Chen, Y., Chou, K., El-Sheikh, A., and Buelow, R. (2002). Orally administered RDP58 reduces the severity of dextran sodium sulphate induced colitis. Ann. Rheum. Dis. 61(Suppl. 2), 19-24. doi: 10.1136/ard.61.suppl_2.ii19

Breiman, L. (2001). Random forests. Mach. Learn. 45, 5-32. doi: 10.1023/A:1010933404324 
Carugo, O. (2013). Frequency of dipeptides and antidipeptides. Comput. Struct. Biotechnol. J. 8:e201308001. doi: 10.5936/csbj.201308001

Centor, R. M. (1991). Signal detectability - the use of roc curves and their analyses. Med. Decis. Making 11, 102-106. doi: 10.1177/0272989X9101100205

Corrigan, M., Hirschfield, G. M., Oo, Y. H., and Adams, D. H. (2015). Autoimmune hepatitis: an approach to disease understanding and management. Br. Med. Bull. 114, 181-191. doi: 10.1093/bmb/ldv021

Delgado, M., and Ganea, D. (2008). Anti-inflammatory neuropeptides: a new class of endogenous immunoregulatory agents. Brain Behav. Immun. 22, 1146-1151. doi: 10.1016/j.bbi.2008.06.001

Ferrero-Miliani, L., Nielsen, O. H., Andersen, P. S., and Girardin, S. E. (2007). Chronic inflammation: importance of NOD2 and NALP3 in interleukin-1beta generation. Clin. Exp. Immunol. 147, 227-235. doi: $10.1111 / j .1365-2249.2006 .03261 . x$

Frank, E., Hall, M., Trigg, L., Holmes, G., and Witten, I. H. (2004). Data mining in bioinformatics using Weka. Bioinformatics 20, 2479-2481. doi: 10.1093/bioinformatics/bth261

Gonzalez, R. R., Fong, T., Belmar, N., Saban, M., Felsen, D., and Te, A. (2005). Modulating bladder neuro-inflammation: RDP58, a novel anti-inflammatory peptide, decreases inflammation and nerve growth factor production in experimental cystitis. J. Urol. 173, 630-634. doi: 10.1097/01.ju.0000143192.68223.f7

Gonzalez-Rey, E., Anderson, P., and Delgado, M. (2007). Emerging roles of vasoactive intestinal peptide: a new approach for autoimmune therapy. Ann. Rheum. Dis. 66(Suppl 3), 70-76. doi: 10.1136/ard.2007.078519

Gribskov, M., and Robinson, N. L. (1996). Use of receiver operating characteristic (ROC) analysis to evaluate sequence matching. Comput. Chem. 20, 25-33. doi: 10.1016/S0097-8485(96)80004-0

Gupta, S., Sharma, A. K., Shastri, V., Madhu, M. K., and Sharma, V. K. (2017). Prediction of anti-inflammatory proteins/peptides: an insilico approach. J. Transl. Med. 15:7. doi: 10.1186/s12967-016-1103-6

Hasan, M. M., Guo, D., and Kurata, H. (2017a). Computational identification of protein S-sulfenylation sites by incorporating the multiple sequence features information. Mol. Biosyst. 13, 2545-2550. doi: 10.1039/C7MB00491E

Hasan, M. M., Khatun, M. S., and Kurata, H. (2018a). A comprehensive review of in silico analysis for protein S-sulfenylation sites. Protein Pept. Lett. 25, 815-821. doi: 10.2174/0929866525666180905110619

Hasan, M. M., Khatun, M. S., Mollah, M. N. H., Cao, Y., and Guo, D. (2017b). A systematic identification of species-specific protein succinylation sites using joint element features information. Int. J. Nanomed. 12, 6303-6315. doi: $10.2147 / \mathrm{IJN}$.S140875

Hasan, M. M., Khatun, M. S., Mollah, M. N. H., Yong, C., and Dianjing G. (2018b). NTyroSite: Computational identification of protein nitrotyrosine sites using sequence evolutionary features. Molecules 23:1667. doi: 10.3390/molecules 23071667

Hasan, M. M., and Kurata, H. (2018). GPSuc:global prediction of generic and species-specific succinylation sites by aggregating multiple sequence features. PLoS ONE 13:e0200283. doi: 10.1371/journal.pone.0200283

Hasan, M. M., Yang, S., Zhou, Y., and Mollah, M. N. (2016). SuccinSite: a computational tool for the prediction of protein succinylation sites by exploiting the amino acid patterns and properties. Mol. Biosyst. 12, 786-795. doi: 10.1039/C5MB00853K

Hasan, M. M., Zhou, Y., Lu, X., Li, J., Song, J., and Zhang, Z. (2015). Computational identification of protein pupylation sites by using profilebased composition of k-spaced amino acid pairs. PLoS ONE 10:e0129635. doi: 10.1371/journal.pone.0129635

Hearst, M. A. (1998). Support vector machines. IEEE Intell. Syst. 18-28. doi: $10.1109 / 5254.708428$

Hernández-Flórez, D., and Valor, L. (2016). Protein-kinase inhibitors: a new treatment pathway for autoimmune and inflammatory diseases? Reumatol. Clin. 12, 91-99. doi: 10.1016/j.reuma.2015.06.004

Huang, S. H. (2015). Supervised feature selection: a tutorial. Artif. Intell. Res. 4:6. doi: 10.5430/air.v4n2p22

Huang, Y., Niu, B., Gao, Y., Fu, L., and Li, W. (2010). CD-HIT Suite: a web server for clustering and comparing biological sequences. Bioinformatics 26, 680-682. doi: 10.1093/bioinformatics/btq003

Ialenti, A., Santagada, V., Caliendo, G., Severino, B., Fiorino, F., Maffia, P., et al. (2001). Synthesis of novel anti-inflammatory peptides derived from the aminoacid sequence of the bioactive protein SV-IV. Eur. J. Biochem. 268, 3399-3406. doi: $10.1046 / j .1432-1327.2001 .02236 . x$
Jin, Y., Wi, H. J., Choi, M. H., Hong, S. T., and Bae, Y. M. (2014). Regulation of anti-inflammatory cytokines IL-10 and TGF-beta in mouse dendritic cells through treatment with Clonorchis sinensis crude antigen. Exp. Mol. Med. 46:e74. doi: 10.1038/emm.2013.144

Kawashima, S., Pokarowski, P., Pokarowska, M., Kolinski, A., Katayama, T., and Kanehisa, M. (2008). AAindex: amino acid index database, progress report 2008. Nucleic Acids Res. 36, D202-D205. doi: 10.1093/nar/gkm998

Kempuraj, D., Selvakumar, G. P., Thangavel, R., Ahmed, M. E., Zaheer, S., Raikwar, S. P., et al. (2017). Mast cell activation in brain injury, stress, and post-traumatic stress disorder and alzheimer's disease pathogenesis. Front. Neurosci. 11:703. doi: $10.3389 /$ fnins.2017.00703

López, Y., Sharma, A., Dehzangi, A., Lal, S. P., Taherzadeh, G., Sattar, A., et al. (2018). Success: evolutionary and structural properties of amino acids prove effective for succinylation site prediction. BMC Genom. 19:923. doi: $10.1186 / s 12864-017-4336-8$

Lowd, D. (2005). "Naive Bayes models for probability estimation," in 05 Proceedings of the 22nd International Conference on Machine Learning (New York, NY), 529-536. doi: 10.1145/1102351.1102418

Manavalan, B., Basith, S., Shin, T. H., Choi, S., Kim, M. O., and Lee, G. (2017). MLACP: machine-learning-based prediction of anticancer peptides. Oncotarget 8, 77121-77136. doi: 10.18632/oncotarget.20365

Manavalan, B., Shin, T. H., Kim, M. O., and Lee, G. (2018). AIPpred: sequencebased prediction of anti-inflammatory peptides using random forest. Front. Pharmacol. 9:276. doi: 10.3389/fphar.2018.00276

Marie, C., Pitton, C., Fitting, C., and Cavaillon, J. M. (1996). Regulation by anti-inflammatory cytokines (IL-4, IL-10, IL-13, TGFbeta)of interleukin-8 production by LPS- and/ or TNFalpha-activated human polymorphonuclear cells. Med. Inflamm. 5, 334-340. doi: 10.1155/S0962935196000488

Michalski, R. S., Carbonell J. G., Mitchell T. M. (2013). Machine Learning: An Artificial Intelligence Approach. Berlin; Heidelberg: Springer-Verlag. doi: 10.1007/978-3-662-12405-5

Miele, L., Cordella-Miele, E., Facchiano, A., and Mukherjee, A. B. (1988). Novel anti-inflammatory peptides from the region of highest similarity between uteroglobin and lipocortin I. Nature 335, 726-730. doi: 10.1038/335726a0

Patterson, H., Nibbs, R., Mcinnes, I., and Siebert, S. (2014). Protein kinase inhibitors in the treatment of inflammatory and autoimmune diseases. Clin. Exp. Immunol. 176, 1-10. doi: 10.1111/cei.12248

Steinman, L., Merrill, J. T., Mcinnes, I. B., and Peakman, M. (2012). Optimization of current and future therapy for autoimmune diseases. Nat. Med. 18, 59-65. doi: $10.1038 / \mathrm{nm} .2625$

Tabas, I., and Glass, C. K. (2013). Anti-inflammatory therapy in chronic disease: challenges and opportunities. Science 339, 166-172. doi: $10.1126 /$ science. 1230720

Vita, R., Mahajan, S., Overton, J. A., Dhanda, S. K., Martini, S., Cantrell, J. R., et al. (2019). The immune epitope database (IEDB): 2018 update. Nucleic Acids Res. 47:D339-D343. doi: 10.1093/nar/gky1006

Yang, Y., Heffernan, R., Paliwal, K., Lyons, J., Dehzangi, A., Sharma, A., et al. (2017). SPIDER2: a package to predict secondary structure, accessible surface area, and main-chain torsional angles by deep neural networks. Methods Mol. Biol. 1484, 55-63. doi: 10.1007/978-1-4939-6406-2_6

Zhao, L., Wang, X., Zhang, X. L., and Xie, Q. F. (2016). Purification and identification of anti-inflammatory peptides derived from simulated gastrointestinal digests of velvet antler protein (Cervus elaphus Linnaeus). J. Food Drug. Anal. 24, 376-384. doi: 10.1016/j.jfda.2015.10.003

Zouki, C., Ouellet, S., and Filep, J. G. (2000). The anti-inflammatory peptides, antiflammins, regulate the expression of adhesion molecules on human leukocytes and prevent neutrophil adhesion to endothelial cells. FASEB J. 14, 572-580. doi: 10.1096/fasebj.14.3.572

Conflict of Interest Statement: The authors declare that the research was conducted in the absence of any commercial or financial relationships that could be construed as a potential conflict of interest.

Copyright (c) 2019 Khatun, Hasan and Kurata. This is an open-access article distributed under the terms of the Creative Commons Attribution License (CC BY). The use, distribution or reproduction in other forums is permitted, provided the original author(s) and the copyright owner(s) are credited and that the original publication in this journal is cited, in accordance with accepted academic practice. No use, distribution or reproduction is permitted which does not comply with these terms. 